\title{
DIE FRAGE DER TERRITORIALEN SOUVERAENITAET ÜBER DIE FALKLANDINSELN
}

\author{
Dr. A, Füsun ARSAVA
}

Der Krieg um die Falklandinseln, ausgelöst durch die argentinische Invasion vom 2. April 1982 und die darauf erfolgte militärische Reaktion der Engländer, hat in der bedingungslosen Kapitulation der argentinischen Streitkräfte am 14. Juli ein Ende gefunden. Jedoch der zwischen Grossbritannien und Argentinien strittige Anspruch auf die Hoheitsgewalt über den Archipel bildet weiterhin den Gegenstand der Auseinandersetzung. Eine Klärung der Frage der Souveränität über die Falklandinseln scheint heute weiter entfernt denn je.

Daher sollen im Folgenden die Ansprüche beider Staaten untersucht werden, wobei die geschichtlichen Fakten, soweit sie entscheldungserheblich sind und die Grunalage für einen Rechtstitel bilden könnten, Darstellung finden.

Dabei ist zu berücksichtigen, dass nach den Regeln des 'intertemporalen Rechts' jedes geschichtliche Ereignis, dass für' die Entstehhung eines Anspruchs bedeutsam ist, nach jenem Recht $\mathrm{zu}$ beurteilen ist, das zur Zeit des Geschehnisses galt und nicht nach den Normen, die zu dem Zeitpunkt in Kraft sind, wenn die Entstehung des Anspruchs Gegenstand eines Rechtsstreits wird ${ }^{1}$.

I. Die Entdeckung der Inseln im 16. jahrhundert und ihre Rechtserheblichkeit.

Der Entdeckung der Inseln wird von verschiedenen Nationen für mehrere Seefahrer in Anspruch genommen, so z. B. soll 1592 John Davis mit dem Schiff 'Desire', 1594 Richard Hawkins mit der 'Dainty' und mit grösserer Sicherheit 1600 Sebald de Weert mit seinem Schiff 'Geloof' als erster die Inseln ge'sichtet haben. Verschiedentlich wird die Entdeckung auch bereits Amerigo Vespucci (1503/4) oder Magellan (1520) zugeschrieben.

${ }^{1}$ Max Huber, Palmas Islands Case, Schiedsspruch des IGH, Zä̈RV 1929 Teil 2, S. 3, ff. (24). 
Im Jahre 1690 erreichte der britische Seefahrer Jonh Strong die Inseln, benannte sie nach dem damaligen Marineschatzmeister Viscount Falkland und ging als Erster hier für einige Tage an Land. Im Verlauf der folgenden Jahre haben häufiger Schiffe verschiedener Nationalität vor den Inseln geankert auf ihren Reisen um Kap Hoorn, jedoch handeite es sich jeweils um nur kurze Aufenthalte, so waren die Inseln z. B. auch französischen Seefahrern bekannt, die sie hach ihrem Heimathafen St. Malo 'Malouines' nannten.

In der Völkerrechtslehre des 17. u. 18. Jahrhunderts herrschte basierend auf dem Begriff der römisch - recht- lichen 'occupatio' die Auffassung vor, der rechtswirksame Gebietserwerb eines Territoriums erfordere zunächst die Freiheit von jeglichem Fremdbesitz, sowie die reale Besitznahme, wobei nicht die Eroberung als solche legitimierend war, sondern die mit Besitzwillen (animus occupandi) ausgeübte andauernde tatsächliche Gewalt im Auftrag der jeweiligen Regierung. Diese Rechtsauffassung teilten mit leichten Modifizierungen alle europäischen Mächte der Zeit. Damit wandte sich die Rechtsauffassung von einem reinen de-iure Besitz, gegründet auf vorherige Zuwendung durch eine päpstliche Bulle (z. B. die Bulle Papst Alexanders VI über die Teilung des Atlantiks von Pol zu Pol 1493) und fiktiven Besitzerwerb durch Eintragung in Karten etc. oder Verträge, ab und dem de facto Besitz zu, der den Gebietserwerb auf das Verhältnis der Möglichkeit tatsächlichır Machtausübung reduzierte.

Daraus folgt, dass abgesehen davon, dass der tatsächliche Entdecker der Inseln nur schwerlich wird festzustellen sein, die blosse Entdeckung als solche noch keinen Rechtstitel, sondern allenfalls eine Anwartschaft auf nachfolgende Besiedlung in angemessener Zeit zu begründen vermag, die ihrerseits jedoch einer später tatsächlich ausgeübten Herrschaft nicht entgegengehalten werden $\mathrm{kann}^{2}$. Es ist mithin davon auszugehen, dass die Inseln bis in die Mitte des 18. Jahrhunderts ein herrenloses Gebiet waren.

II. Die erste Besiedlung durch Frankreich und Aibtretung der Inseln an Spanien 1766.

Die genannten Vorraussetzungen einer rechtsgültigen Besitznahme erfullte erstmals Frankreich, als 1 1764 Louis de Bougain-

${ }^{2}$ Hermann Weber, Falkland-Islands oder Malvinas? Der Status der FalklandInseln im Streit zwischen Grossbritannien und Argentinien, Frankfurt a. M. 1977, S. 60, f.; Max Huber, a.a.O. S. 52. 
ville in Begleitung von Siedlern, die ihr Land in Folge der Verluste im französisch-britischen Kolonialkrieg von 1763 verloren hatten, auf den Inseln landete und hier mit Billigung und im Auftrag seiner Regierung eine Niederlassung gründete, den Hafen St. Louis auf OstFalkland, die binnen Jahresfrist von 150 französischen Siedlern bewohnt wurde.

Ab diesem Zeitpunkt unterlagen die bis dahin eine 'terra nullius' bildenden Inseln der französischen Herrschaftsgewalt.

Spanien wandte sich im Jahre 1765 mit Protestan an den französischen Hof, weil es durch die französische Präsenz seine Stellung auf dem südamerikanischen Kontinent gefährdet sah. Unter dem Druck dieser Proteste schloss Frankreich mit Spanien 1766 einen Vertrag, in dem es sich bereit erklärte, Letzterem gegen Zahlung einer Entschädigungssumme alle Rechte an den Inseln zu übertragen.

Die offizielle Besitzübergabe an Spanien erfolgte im April 1767, worauf Spanien die Siedlung unter dem Namen 'Puerto de la Soledad' weiterführte.

Es hat also eine wirksame Rechtsnachfolge Spaniens in die Souveränitätsrechte Frankreichs stattgefunden.

III. Das Eintreffen der Englaender auf dem Archipel und ihre Vertreibung durch die Spanier

In kurzem zeitlichen Abstand zuder geglückten;Besiedlung Bougainvilles entsandte die englische Krone zwei Schiffe unter dem Befehl Kapitän Byrons zu den Inseln, die sie nach den Beschreibungen älterer Seefahrer als Schlüssel zum gesamten Pazifik betrạchteten, ohne von der bereits erfolgten Inbesitznahme durch die Franzosen Kenntnis zu haben. Vielmehr hielt man die Inseln für von Menschen unberührt und beeilte sich daher eine Expedition auszurüsten. Im Januar 1765 landete Byron auf einer der westlichen Inselgruppe angehörenden Insel, nahm diese und den gesamten Archipel formell für den englischen König in Besitz, hinterliess einige englische Hoheitszeichen und kehrte mit Berichten nach hause zurück, die die Admiralität veranlassten Kapitän John McBride mit der Errichtung einer Garnison auf den nach wie vor für unbewohnt gehaltenen Inseln zu beauftragen. Dieser traf im Januar 1766 am Ort des Geschehens' ein und begann mit der Besiedlung der westlichen der beiden Inseln Die französische Ansiedlung entdeckte McBride erst im, Dezember desselben Jahres anlässlich einer Erkundungsfahrt. Beide Seiten protestierten unver- 
züglich gegen die Anwesenheit des Anderen unter Berufung auf ihre Rechte, was jedoch zunächst an der Situation nichts änderte, beide Siedlungen bestanden unbeeinträchtigt nebeneinander fort.

Im April 1767 erfolgte der vertragliche Übergang der französischen Rechte an Spanien und erst Ende 1769 erhob der spanische Befehlshaber der Inseln Proteste gegen die britische Präsenz. Diese blieben genau wie nachfolgende erfolglos, worauf im Juni 1770 eine Übermacht spanischer Schiffe in den Hafen der Engländer einlief, diese zur Kapitulation und zum Verlassen der Inseln zwang und die englische Siedlung zerstörte.

IV. Hat England einen Konkurrierenden Rechtstitel erworben?

Es stellt sich nun die Frage, ob die Engländer durch ihre Aktion gleichfalls einen Rechtstitel erlangt hatten. Wie oben dargestellt, haben bereits ab 1764 die Handlungen Bougainvilles den Vorraussetzungen einer rechtsgültigen Okkupation entsprochen. England, das wenn auch nur kurze Zeit später, auf dem Archipel erschien, konnte somit keinen Rechtserwerb durch Okkupation mehr tätigen, denn, gleichgültig, ob man die Expedition Byrons oder McBrides als erfolgreiche Besiedlung betrachtet, so fehlte doch in jedem Fall zu diesem Zeitpunkt dem Gebiet die Eigenschaft als 'terra nullius'. Es konnte also in keinem Fall ein Rechtserwerb Englands an dem gesamten Archipel mehr eintreten. An diesem Tatbestand ändert auch die Zession der französischen Rechte an Spanien nichts, da Spanien hierdurch wirksam in die Rechtsposition Frankreichs cintrat, unter Wahrung der Priorität seiner Ansprüche gegenüber denen Englands. In der Folgezeit hat Spanien die Besitzung in einer dem Prinzip der Effektivität der Herrschaftsausübung genügenden Weise fortgeführt, so dass sein Titel auch nicht nach der Übernahme unterging.

Man kann sich allerdings fragen, ob nicht für England und Frank-. reich bzw. Spanien jeweils ein teilweiser Gebietserwerb stattgefundén hat, in den Grenzen des von ihnen tatsächlich besessenen Gebiets. Diese Hypothese kann aber wohl nach dem Willen der Staaten, die die Inseln als einheitliches Gebiet betrachteten, ausgeschlossen werden. Dieses umso mehr, als auch die Besetzung eines Teiles des Gebietes den Anforderungen der Okkupation durch effektive Besitznahme genügt, da insoweit nicht die tatsächliche Beherrschung jedes Landstücks, die auch nach den geographischen und klimatischen Bedin-

${ }^{3}$ anders wohl nur Metiord J.C.J. Falklands or Malvinas, the Background of the Dispute, International Affairs 1968, vol.. 44, S. 463, ff. (470). 
gungen kaum verlangt werden kann, sondern vielmehr die potentielle Beherrschbarkeit, d.h. auch Verteidigungsfähigkeit ausschlaggebend ist. Der englische Besitz stelit also keinen Rechtstitel, sondern nur einen de-facto Besitz dar, der spätestens seit En'tdeckung der französischen Siedlung und Kenntnisnahme von deren' älteren Rechten lllegitim aufrechterhalten wurde.

Die Vertreibung der Engländer durch die Spanier war folglich nicht rechtswidrig im Sinne des intertemporalen Rechts, sondern die legitime Wiederherstellung ihrer uneingeschränkten Rechtsposition, die auch mit Waffengewalt geschehen durfte, zumal vorhergehende Aufforderungen und Proteste erfolglos blieben ${ }^{4}$.

V. Der english-spanische Kompromiss von 1771 und seine rechtliche Bedeutung

Die Ereignisse des Jahres 1770 entfesselten eine schwere diplomatische Krise, die England und Spanien an den Rand eines Krieges brachte, den zu führen beide Staaten weder vorbereitet noch willens waren. In langandauernden Verhandlungen wurde von dem spanischen und dem britischen Botschafter ein Vertrag beschlossen und am 22.1.1771 unterzeichnet, der den Engländern Satisfaktion für die gewaltsamen Übergriffe der Spanier zusicherte, die Wiederherstellung des vorher bestehenden Zustandes und Übergabe von Port-Egmont an die Briten garantierte. Die Rechtsposition der, Englaender findet in der spanischen Vertragserklärung keine Erwähnung, viel mehr behält sich Spanien die Souveränität über die gesamten Inseln vor. Die englische Vertragserklärung bestätigt die spanischen Aussagen unter Ignorierung des spanischen Souveränitätsvorbehalts, ohne jedoch auch eigene Territorialanısprüche niederzulegen ${ }^{5}$.

Vielfach wird behauptet, Spanien habe sich zum Abschluss dieses Vertrages durch geheime Botschaften bzw. mündiịche Zusagen der Engländer bewegen lassen, die Inseln nach Gewährung der Satisfaction zu raeumen ${ }^{6}$. Ob dies zutrifft, kann nicht bewiesen und somit der Beurteilung des Vertrages auch nicht zu Grunde gelegt werden.

Dieses Abkommen ist sehr unterschiedlich interpretiert worden, wobei die extremen Positionen die Annahme einer Anerkennung der spanischen Souveränität durch England bzw. den umgekehrten Fall

\footnotetext{
H. Weber., a.a.O., S. 65.

s Text abgedruckt bei Hillekamps, Der Streit um die Falklañd-inseln, Diss, Köln. 1878, S. 83., f.
} 
bilden. Eine vorsichtige Auslegung des Vertrags-textes legt jedoch andere Schlüsse nahe. An keiner Stelle des Textes wird Bezug auf englische Rechte oder die Verletzung derselben genommen, vielmehr soll die Wiedergutmachung einer erlittenen Ehrverletzung der englischen Krone durch die Spanier bewirkt werden. Auf der anderen Seite schweigt England ebenfalls bezüglich der spanischen Souveränitätsrechte, widerspricht diesen aber auch nicht. IMan muss daher davon ausgehen, dass den Parteien nicht vorwiegend an einer Klärung der Souveränitätsfrage, sondern zunächst an der Herstellung des 'status quo ante' gelegen war um weitere Gewaltakte zu verhindern und man die Rechtslage zunächst auf sich beruhen liess.

Nichtsdestotrotz wurde England durch den Vertrag und die im September 1771 erfolgte Übergabe die völkerrechtlich bedeutsame Möglichkeit eingeräumt die tatsächliche Herrschaftsgewalt auf den Falkland Inseln zu erneuern und auszuüben, die nach intertemporalem Recht durch langandauernde effektive Herrschaftsausübung zum Gebietserwerb haette erstarken können?, zumal die Frage der Souveränität zwischen beiden Staaten nicht präjudiziert war ${ }^{8}$.

\section{Der Rückzug der Englaender im Jahre 1774.}

Im Mai 1774, drei Jahre nach den spanisch-britischen Kompromiss räumten die Engländer : indessen Port Egmont und verliessen die Inseln im Zuge von Einsparungsmassnahmen, jedoch unter Zurücklassung einer Bleiplatte, auf der die Rechte der alleinigen Souveränität über den Archipel dem englischen König vorbehalten würden.

Dieser Rückzug der Engländer erfolgte aus freier unbeeinflusster Entscheidung, ohne Bezug auf den zuvor geschlossenen Vertrag?. Durch diese Aufgabe des de facto Besitzes ging England der Möglichkeit verlustig, durch Ausübung langandauernden Besitzes gegründet auf das Effektivitätsprinzip auch de iure Besitz zu erwerben und stellte damit die uneingeschränkte legitime Gebietshoheit Spaniens widerher.

Da England wie dargestellt weder durch originären Erwerb, noch durch Vertrag bis zu diesem Zeitpunkt Souveränitätsrechte erworben

6 heimlich deswegen um die Opposition im engl. Parlament u. die Öffentlichkeit nicht zuerregen.

7 Seidl-Hohenfeldern Ignaz, Völkerrecht, 4. Aufl. Berlin, 1980., Rdn. 123 of.

- anderer Ansicht ist offensichtlich Blumenwitz Dieter, Zeitschrift für Politik 1982, S. 318., ff. (324).

9 wie, oben bereits erwaehnt sind angebliche englische. Zusicherungen des Rückzugs nicht nachzuweisen $u$. auf jeden Fall nicht Gegenstand des Vertrages geworden. 
hatte, konnte es sich diese auch nicht durch Hinterlassung von den Souveränitätsanspruch ausdrückenden Smbolen sichern. Darüber hinaus ist die Reservierung auch rechtmässig erworbener Ansprüche durch symbolische Hoheitszeichen nur sehr begrenzt möglich, da wie oben bereits angesprochen zum rechtsgültigen Erwerb eines Titels stets die effektive Ausübung von Hoheitsgewalt trèten muss.

Insoweit ist auch hier die Frage der Dereliktion des Territoriums durch England nicht einschlägig ${ }^{10}$, denn England verfügte über keine gєsicherte Rechtsposition, die es hätte aufgeben können, sondern begab sich lediglich seiner Anwartschaft auf künftigen Rechtserwerb.

Zusammendfassend ist festzustellen, dass Spanien nach dem Abzug der Englaender im Jahre 1774 alleiniger rectmaessiger Inhaber des territorialen Souveränität und Gebietshoeit über die Falkland Inseln war.

VII. Die geschichtliche Entwicklung der Inseln bis 1832.

In den folgenden Jahren übte Spanien seine Herrschaft unangefochten aus, liess 1777 die Überreste der englischen Ansiedlung endgültig zerstören um etwaigen späteren Konflikten mit England vorzubeugen, baute die Hoheitsbefugnisse seiner Verwaltung über die Inseln aus und nützte diese vorwiegend als Strafkolonie., Im Jahre 1811 entschied der spanische Vizekönig, bedrängt durch die seit 1810 aufbegehrenden Revolten gegen das spanische Mutterland, die Garnison aus Puerto de la Soledad abzuziehen und die Siedler auf das Festland Patagoniens zu evakuieren. Diese Massnahme wurde von Spanien als provizorische bis zur erneuten Befriedung der südamerikanischen Besitzungen deklariert. Der Unabhängigskeitskrieg des Vizekönigreichs Buenos Aires, zu dem die Inseln gehörten, endete indes mit der Unabhangigkeitserlärung der "Provincias Unidas del Rio de la Plata" am 9. Juli 1816, und Spanien hat bis in die Gegenwart niemals mehr ein ausdrückliches Interesse an den Inseln bekundet. 'Die folgenden Jahren waren bestimmt vom Kampf der jungen Republik, die zunächst nur von den U.S.A. informell anerkannt wurde, um inneren Zusammenhalt und gegen äussere Bedrohung.

In dem Bemühen die Kontrolle über alle der ehemals Spanien unterstellten Gebiete zu gewinnen, entsandte die Reglerung in Buenos Aires erst mals 1820 einen Vertreter der die Inseln formell für die Republik in Besitz nahm, die Flagge der Republik in Puerto de la Soledad hisste, ein Jagd- und Fischereiverbot für die Inseln erliess und

10 so aber Blumenwitz a.a.O., S. 324. 
dieses, sowie die Besitzergreifung den Kapitänen der umliegend ankernden Schiffe bekanntgab. Darauf kehrte er nach Buenos Aires züruck.

1823 erfolgte die formelle Anerkennung der Republik durch Amerika. Im selben Jahr wurde den ersten Gouverneur für die Inseln bestimmt und an "Louis Vernet" Konzessionen zur Fischerei und Viehzucht auf Ostfalkland vergeben. Der erste Versuch Vernet's zum Aufbau einer Kolonie schlug allerdings fehl, so dass er 1826 eine zweite, diesmal erfolgreiche Anstrengung zur Besiedlung mit hundert Menschen, ausgestattet nunmehr mit exklusiven Konzessionen für die gesamten Inseln, unternahm.

Dies alles geschah mit Kenntnis der englischen Krone die 1825 die Republik in einem Freundschafts- und Handelsvertrag formell anerkannte.

Die Kolonie Vernets entwickelte sich in der Folge so vielversprechend, dass er 1829 zum 'Gobernador Civil y Militar de las Islas Malvinas y sus Adyacencias' ernannt wurde, was England am 19.11.1829 zu formellen Protesten unter Berufung auf ihre angeblichen älteren Rechte an den Inseln veranlasste.

Im Juli 1831 kam es zu Zwischenfällen mit amerikanischen Seeleuten, die die von Vernet in seiner Eigenschaft als Gouverneur orneuerten Jagd- $\mathrm{u}$. Fischereiverbote missachtet hatten, in deren Verlauf Vernet ein amerikanisches Schiff aufbrachte und zur gerichtlichen Klärung des Vorfalls nach Buenos Aires überführte. Als amerikanische Proteste gegen dieses Vorgehen und die Forderung nach sofortiger Freigabe des Schiffs ohne Erfolg blieben, ordnete die amerikanische Regierung im Dezember 1831 die Entsendung des Kriegsschiffes 'Lexington' auf die Inseln an, wo der Kapitän die Vertreter Vernets festnehmen liess, die argentinischen Verteidigungsanlagen zerstörte, die Inseln plünderte und die Bewohner Soledads unter Arrest setzte. Anschliessend erklärte er die Inseln als "free of all government" und kehrte mit seinen Gefangenen zurück.

In den nachfolgenden schweren diplomatischen Auseinandersetzungen stellte sich England auf die Seite der USA, indem es die argentinische Handlungsweise als Akt der Piraterie und Usurpation britischer Hoheitsrechte qualifizierte.

VIII. Die rechliche Beurteilung der Geschnisse

Es fragt sich, wie diese Geschehnisse rechtlich zu beurteilen sind 
und insbesondere, ob Argentinien nach dem Rückzug der Spanier von den Inseln einen rechtsgültigen Titel erlangt hat.

\section{Die Dereliktion der Inseln durch Spanien}

Zunächst ist daher also zu klären, ob Spanien durch seinen Rück. zug 1811 oder zu einem späteren Zeitpunkt seine Souveränitätsrechte an den Inseln verloren hat.

In der Räumung der Inseln durch die Spanịer könnte eine Dereliktion des Territoriums liegen. Davon ist auszugehen, wenn zu der tatsächlichen Aufgabie eines Gebietes der manifestierte Wille des Gebietsherrn tritt, auf die Ausübung der Hoheitsgewalt endgültig zu verzichten. Allerdings kann eine solche Absicht auch den Umständen entnommen werden ${ }^{11}$. Bedenken hinsichtlich eines Dereliktionswillens der Spanier könnten sich daraus ergeben, dass die spanische Regierung anlässlich der Räumung die Absicht kundgegeben hat auf die Inseln zurückzukehren. Dieser Erklärung sind jedoch niemals Taten gefolgt, noch hat Spanien jemals, wieder ein Interesse an den Inseln gezeigt.

Weiterhin hat die argentinische Besiedlung erst nach einer längeren Zeitspanne $(1826=15$. Jahre $)$ stattgefunden,; während derer die aufständischen Provinzen zur Unabhängigkeit gelangten und eine Konsolidierung der jungen Republik durch begrenzte Anerkennung und effektive Hoheitsausübung sich vollzog. Damit konnte schon zu diesem Zeitpunkt der spanische Rückzug nicht mehr als bloss vorübergehende Massnahme betrachtet werden.

Dasentscheidende Indiz für eine Dereliktionsábsicht ist jedoch, dass Spanien zu keiner Zeit gegen die Besetzung der Inseln protestiert hat. Nicht gegen die der Argentinier, was unter Umständen als defacto Anerkennung der Republik hätte ausgelegt werden können, aber auch nicht gegen die der Engländer. Das bedeutet aber implizit, dass es sich bereits mit einem Verlust der territorialen Souveränität vor dem Erscheinen der Engländer und zwar an die 'Provincias Unidas' abgefunden hatte und deren Besetzung nicht bloss als eine Aktion einer seiner territorialen Untergliederungen betrachtete ${ }^{12}$.

Unter Berücksichtigung der an gefürten Umstände ist davon auszugehen, dașs Spanien in den Jahren nach dem: Rückzug bis zur

"Verdross Alfred-Simma Bruno., Universelles Völkerrecht, Theorie und Praxis 2. Aufl. Berlin, 1980., S. 558.

12 insofern fehlgehend meiner Ansiçht nach die Argumentation H. Webers, a.a.O.

s. 71-76, nach der Spanien bis zur Anerkennung der Republik 1858 seinen Eigentumsanpruch auf die Inseln aufrechterhalten hat. 
argentinischen Besetzung die Inseln mit animus derelinquendi aufgegeben hat.

\section{Hat eine Rechtsnachfolge der "Provinciales Unidas" stattge- funden.}

Die 'Provincias Unidas' könnten einen Titel zumeinen durch Rechtsnachfolge in die Rechtspositionen Spaniens erlangt haben.

Aber für eine vertragliche Zession der Rechte besteht kein Anhaltspunkt und von einer stillschweigenden Übertragung ist ebenfalls nicht auszugehen, insbesondere, wenn man berücksichtigt, dass Spaninen die Republik erst 1858 anerkannt hat.

\section{Ergibt sich aus der "Uti possidetis" Doktrin ein Anspruch?}

Argentinien könnte eventuell einen Rechtsanspruch aus der 'uti possidetis' Doktrin herleiten.

Dieses Prinzip, das zum Teil von den neugebildeten Latein-amerikanischen Staaten als Verfassungsprinzip übernommen wurde, besagi, dass die Grenzen der spanischen gewordenen Republiken mit denen der ehemaligen spanischen Provinzen übereinstimmen sollen, so dass auch die noch nicht tatsächlich in Besitz genommenen Territorien de iure bereits zum Gebiet des Nachfolgestaats gehören sollten, mit dem Effekt, dass auch nach dem Untergang des spanischen Kolonialreichs keine herrenlosen Territorien auf dem südamerikanischen Kontinent existieren würden.

Dieses Prinzip diente zum einen der Sicherung der Grenzen der neu entstandenen Staaten gegeneinander, wie auch der Abwehr erneuter Kolonialisierung durch europäische Staaten. Danach würden alsò die Falklandinseln automatisch den 'Provincias Unidas' zugefallen sein, da sie vormals zum Vizekönigreich Buenos Aires gehörten.

Allein ist die Anwendung dieses Prinzips auf den vorliegenden Fall verschiedenen Bedenken ausgesetzt. Von einigen Autoren wird vor allem die Uneinheitlichkeit der Auslegung der Dokktrin bemängelt, wobei insbesondere nicht eindeutig ist, ob das 'uti possidetis' de iure oder de facto ausschlaggebend ist ${ }^{13}$. Ferner wird der Widerspruch $\mathrm{zu}$ dem allgemeinen völkerrechtlichen Grundsatz der effektiven Okkupation kritisiert, der einen blossen de iure Besitzerwerb nicht genügen

${ }^{13}$ Strupp-Schlochaver, Wörterbuch des Völkerrechts, Bd. III, Berlin, 1961, S. 483 
lässt und daraus ein möglicher Vorrang des allgemeinen Völkerrechtabgeleitet ${ }^{14}$.

Das entscheidende Argument scheint jedoch zu sein, dass wegen der Entwicklung und ausschliesslichen Anwendung dieses Prinzips auf dem Jateinamerikanischen Kontinent, es sich hierbei um eine regional begrenzte Norm partikularen Völkerrechts handelt Grossbritannien als ein ausserhalb dieser Region stehender Dritter braucht sich deshalb die 'uti possidetis' Doktrin nicht éntgegenhalten zu lassen.

\section{Der Okkupationserwerb durch Argentinien '}

Schliesslich könnten die 'Provincias Unidas' einen Anspruch durch effektive Okkupation eines herrenlosen Gebiets erworben haben.

Wie bereits oben dargelegt, sind die Inseln durch spanische Dereliktion $\mathrm{zu}$ einer terra nullius geworden. Es müsste also das Kriterium der von Besitzwillen getragenen, dauernden und effektiven Ausübung von Hoheitsgewalt gegeben sein. Die 1820 erfolgte; formelle Besitznahme der Inseln, die sich vorwiegend auf symbolische Hoheitsakte erstreckte, wird diesen Anforderungen genau so wenig gerecht, wie die erste fruchtlose Expedition Vernets von 1823. Dagegen bildet der zweite, erfolgreiche Versuch Vernets mit der Ansiediung von hundert Menschen nicht nur eine tatsächliche Besitznahme, sondern durch die Einsetzung eines Zivil- und Militärgouverneurs un'd Stationierung einer Garnison, auch die Ausübung staatsgetragener Hoheitsgewalt mit animus occupandi.

Die äussere und innere instabile Situation der jungen Republik hatte auf den Rechtserwerb keinen Einfluss, da sie zum einen bereits beschränkte Anerkennung gefunden hatte, sowie zưr Setzung von Akten staatlicher Hoheitsgewalt in der Lage war ${ }^{15}$. Auch die seit 1829 nach Einsetzung der Gouverneurs und in Zusammenhang mit der 'Lexington Affaire' vorgetragenen britischen Proteste'vermochten den Rechtserwerb nicht zu hindern, da wie bereits dargestellt, Grossbiritannien keinerlei Rechte an den Inseln besass. Darüber hinaus hätte diesen Einwänden die Einrede des 'venire contra factum propium' (Estoppel) entgegen gehalten werden können, da 'Grossbritannien bereits 1823 von der Verteilung der Landkonzessionen an Louis Vernet Kenntnis genommen hatte.

14 Hillekamps, a.a.O. S. 115.

15 siehe insoweit auch das Zitat des amerikanischen Vertreters Poinsett, abgedruckt bei H. Weber, a.a.O. S. 84 . 


\section{Die rechtlichen Auswirkungen der "Lexington Affaire"}

Es fragt sich, ob das Recht der 'Provincias Unidas' durch die Vertreibung der Siedler in der "Lexington Affaire" untergegangen ist.

Es scheint nicht einwandfrei geklärt, ob nach der vollständigen Vernichtung des Gegners in lediglich einem Teil seines Gebietes, nach intertemporalen Recht der Sieger berechtigt war, dieses als terra nullius anzugeben, wie es durch den Kapitän der Lexington geschehen ist.

Berücksichtigt man die in der Folge unverzüglich wieder-aufgenommenen argentinischen Besiedlungsanstrengungen, sowie die später neutrale Haltung der USA bezüglich der Souveränitätsfrage ${ }^{16}$, so stellt sich die Vertreibung der Siedler bloss als kurze unschädliche Unterbrechung der Ausübung der Hoheitsgewalt dar, die den vom Besitzwillen getragenenen Rechtsanspruchs nicht untergehen liess.

Ab'schliessend ist also feszustellen, dass die 'Provincias Unidas' nach Dereliktion durch die Spanier mit der Besiedlung von 1829 die territoriale Souveränität über die Falkland Inseln erworben hatte.

\section{Die Rückkehr der Englaender auf die Inseln 1832.}

Noch während die Lexington Affaire in Buenos Aires ihren weiteren Verlauf nahm, wurde Ende des Jahres 1832 von der argentinischen Regierung ein neuer Gouverneur für die Inseln ernannt und mit dem Wiederaufbau von Kolonie und Garnison beauftragt. Als im Jahre 1833 das englische Kriegsschiff Clio den Hafen Puerto de la Soledad Wiederaufbau von Kolonie und Garnison beauftragt. Als im Jahre erreichte, traf es dort die neuen, Vertreter der argentischen Administration an. Ihnen erklärte der Kommandant der Clio, er sei gekommen um die Inseln fǜr den britischen König im Besitz zu nehmen, forderte sie zum Niederholen der argentinischen Flagge und zum Verlassen der. Inseln auf. Nach anfänglichen Protesten und Widerstand sahen sich die Argentinier genötigt, sich der milıtärischen Übermacht der Engländer zu beugen und zogen sich am 4.1.1833 zurück, nicht ohne vorher symbolisch das Kommando auf einen der verbleibenden Siedler übertragen zu haben.

Auch die Englaender verliessen die Inseln um im folgenden Jahr mit zwei Kriegsschiffen zurückzurkehren und endgültig mit der Kolonisierung und Besiedlung der Inseln $\mathrm{zu}$ beginnen, die äusserst erfolg-

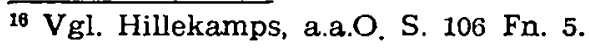


reich verlief, einen Aufbau der Landwirtschaft und des Handels erreichte und ein schnelles Wachstum der Siedlung zur Folge hatte.

Gegen die englische Aktion, die den Besitz Argentiniens an den Falklandinseln bis zum heutigenTag beendete, wurde unverzüglich dem englischen Gesandten in Buenos Aires und am 24.4.1833 u. 17.6. 1833 vom argentinischen Botschafter in London Widerspruch vorgetragen, mit einer Darlegung des argentinischen Rechtsstandpunkts. Die britische Regierung ihrerseits wies alle Proteste : unter Hinweis auf ihre älteren, 1774 erworbenen Souveränitätsrechte zurück und rückte von diesem Standpunkt auch angesichts erneuter Vorbringen des argentinischen Botschafters 184 lund 1849 nicht ab.

In der Zwischenzeit wurde die Kolonisation der Inseln vorangetrieben, 1841 wurde ein Gouverneur eingesetzt und eine dauernde Zivilverwaltung errichtet, durch Gesetz von 1843 wurden die Inseln der englischen Legislative unterstellt und weitere Schritte zur verfassungsrechtzlichen Ausgestaltung unternommen.

Im Jahre 1849 schlossen Argentinien und England einen Freundschaftsvertrag, ohne dass weitere Stellungnahmen zum Souveraenitaetsstreit erfolg't waeren, der erst $1888 \mathrm{zu}$ einem erneuten Notenwechsel führte, als England gegen einen argentinischen Atlas protestierte, der Insel als argentinisches Territorium aúswies. Die Kontroverse lebte erst anlässlich des 'Briefmarkenstreits' 1932 wieder auf, als Argentinien gegen eine britische, den Falklandinseln gewidmete Briefmarkenserie protestierte und 1936 seinerseits eine Briefmarke, auf der die Inseln als argentinisches Gebiet dargestellt wurden herausgab. Von diesem Zeitpunkt an hat Argentinien wiederholt und nachdrücklich seinen Ansprach geltend gemacht und schliesslich den Streit 1964 vor die Vereinten Nationen gebracht.

\section{Hat England durch Ersitzung einen Rechtstitel erworben?}

Könnte England inder Zwischenzeit einen Rechtstitel erlangt haben?

Argentinien hatte, wie oben gezeigt, seit 1829 die territoriale Souveränität über die Inseln inne, somit bildeten diese für England keine dem originären Okkupationsèrwerb freistehende terra nullius. Ferner stand England kein Rechtsanspruch zu, mit dem es die Besetzung hätte begründen können, weitere Rechtfertigungsgründe für die Inbesitznahme sind nicht ersichtlich. Der Akt mit dem England die Gebietshoheit über die Inseln erwarb, war also rechtswidrig. Der unrechtmässige Gebietserwerb England könnte jedoch nachträglich 
durch Zeitablauf legalisiert worden sein, wenn man zu seinen Gunsten eine Ersitzung annehmen könnte.

Dieses Rechtsinstitut, das der im Völkerrecht bedeutsamen normativen Kraft des Faktischen Rechnung trägt ${ }^{17}$, ermöglicht die Legalisierung unrechtmässig, durch Gewalt etc begründeten Besitzes, unter der Vorraussetzung, dass ein Staat die effektive Hoheitsgewalt mit dem Anspruch auf territoriale Souveränität, wobei er hinsichtlich seines Besitzrechtes nicht notwendig gutgläubig zusein braucht $t^{18}$, den Besitz offenkundig, friedlich und unangefochten über einen angemessen langen Zeitraum ausübt ${ }^{19}$.

Es ist zuprüfen, ob diese Bedingungen erfüllt sind. England hat die Inseln, wie schon aus der Kundgabe bei Vertreibung der Argentinier hervorgeht, mit dem Anspruch der territoriale Souverän zu sein, übernommen. Ob es dabei tatsächlich gutgläubig hinsichtlich des Bestehens älterer Rechte war, ist aus den historischen Fakten nicht ohne weiteres zu entnehmen, kann aber hier dahinstehen. In Bezug auf die Offenkundigkeit der Besitzausübung bestehen ebenfalls keine Zweifel, wobei überhaupt ein 'heimlicher' Besitz im Völkerrecht, schwerlich vorzustellen ist ${ }^{20}$. Schwierigkeiten bereiten dagegen die Erfordernisse eines angemessen langen Zeitraums und friedlicher. unangefochtenen Besitzausübung, da für beide eine allgemeingültige Regelung fehlt und diese jeweils nach den Umständen des Einzelfalls zu beurteilen sind.

Nach anfänglichen lebhaften Protesten auf diplomatischer Ebene gegen den englischen Übergriff, hat Argentinien 1849 in einer Note kundgetan, dàss auch für die Zukunft sein Schweigen nicht als Zustimmung dürfte gedeutet werden. In der Folge lebte die Kontroverse 1888 in der Auseinandersetzung um die Atlanten nur noch einmal auf, bis Argentinien anlässlich des Briefmarkenstreits begann, kontinuierlich seinen Anspruch geltend zumachen.

Es fragt sich, ob die Intensität und Beständigkeit der Proteste ausreichend ist, einen englischen Rechtserwerb $\mathrm{zu}$ verhindern.

Ob diplomatische Proteste für sich allein überhaupt geeignet sind, ist in der Doktrin zumindest umstritten ${ }^{21}$ und wohl in Hinblick auf

\footnotetext{
17 Seidl-Hohenveldern., a.a.O. Rdn. 326, der die Ersitzung als allgemeinen Rechsgrundsatz Qualifiziert.

18 Berber Friedrich, Lehrbuch des Völkerrechts Bd. I. 2. Aufl., München, 1975, S. 365.

${ }^{18}$ Max Huber, a.a.O. S. 16f.

${ }^{20}$ siehe aber Johnson, D.H.N. Acquisitive Prescription in international Law B Y I L XXVII, 1950, S. 332 ff. (347).

21 Johnson a.a.O. S. 346; Berbar, a.a.O. S. 366.
} 
andere zur Verfügung stehende Mittel zu beurteilen, wobei eine Gewaltanwendung von dem betroffenen Staat insbesondere nach dem heutigen Völkerrecht wohl nicht erwartet werden muss. Das Auffällige an der argentinischen Haltung ist aber, dass nach der Note von 1849 auch die diplomatischen Proteste enden und auch der in der Atlantenaffaire ausgedrückte Anspruch Argentiniens auf das Territorium sich auf eine innerstaatliche Aktion beschränkte, die allerdings ihrerseits wieder den diplomatischen Protest Englands auslöste.

Ob aber die Erklärung von 1849 ausreichte um die Proteste bis zum 'Briefmarkenstreit' 1932, also über 80 Jahre aufrecht zu erhalten, ist zweifelhaft, insbesondere, wenn man das übrige Verhalten der Republik, nämlich den Abschluss eines Freundschaftspakts und auch sonstige normale, besonders intensive wirtschaftliche Beziehungen $\mathrm{zu}$ England beachtet. Für eine verhäaltnismässig lange Zeitspanne liess Argentinien die Engländer also in ihrem Besitz unangefochten, während derer viele britische Aktivitäten zur Eingliederung der Inseln unternommen wurden, die sich durch hohe Effizienz auszeichneten. Was allgemein ein rascheres Heilen des rechtlichen Mangels bewirkt ${ }^{22}$. An diesem Punkt sollte vielleicht auch berücksichtigt werden, dass die argentinische Hoheitsgewalt selbst nur sehr kurze Zeit und weit weniger wirkungsvoll ausgeübt wurde.

Wenn man alle entscheidungserheblichen Umstände in Betracht zieht, is aber der Schluss zuläessig, dass die argentinischen Proteste weder an Intensität und noch weniger an Beständigkeit geeignet erscheinen, eine Legalisierung des langandauernden, durch hohe Effizienz gekennzeichneten englischen Besitz zu verhindern. Die in den 30 er Jahren einsetzenden häufigen und bis heute anhaltenden Proteste müssen in dieser Hinsicht als verspätet zurückgewiesen werden.

Der ursprünglich unwirksam erworbene britische Besitz an den Falklandinseln ist also nachträglich, auf Kosten des argentinischen Rechtstitels, geheilt worden. Argentinien hat seine 1829 durch Okkupation erworbene Souveränität durch Ersitzung an England verloren ${ }^{23}$.

XI. Kann Argentinien einen Gebietsanpruch aus Kontiguitaet herleiten

Gibt die Kontiguitaetsthese Argentinien einen ' konkurrierenden Gebietsanspruch gegen England?

22 Dahm, Georg. Völkerrecht Bd. I, Stuttgart, 1958. S. 595

23 Diese Frage ist jedoch lebhaft umstritten, zu einem anderen Ergebnis kommt z. B. Hillekamps, a.a.O. S. 148. 
Diese These besagt als Äusprägung des Prinzips der geographischen Nachbarschaft, dass durch die blosse Naehe einer Inseln zum Festland oder, einer anderen Insel, sich die Souveränität dieses benachbarten Staates auch auf die Insel erstrecke. In der Vergangenheit wurden haeufig Gebietsansprüche mit dieser Theorie gerechtfertigt, so auch von Argentinien mit dem Argument, dass die Inseln als Forsetzung des patagonischen Festlandes erschienen.

Inder Völkkerrechtlichen judikatur ${ }^{24}$ und Literatur ${ }^{25}$ wird die Existenz eines solchen Rechts zur Begründung von Gebietsansprüchen jedoch abgelehnt und findet einzig Anerkennung für den Sonderfall der Nutzung des F'estlandsockels durch den Küstenstaat.

Auch aus Kontiguitaet kann Argentinien keinen Rechtstitel herleiten.

XII. Ist England verpfljchtet seinen Titel aufzugeben?

Argentinien hat die Auseinandersetzung um den, von ihm bestrittenen Rechtstitel Englands vor die Vereinten Nationen gebracht, wo es seit 1964 auf der Tagsesordnung der General-versammmlung, sowie des Sonderkommitees für Dekolonialisierung steht.

Für eine endgültige Entscheidung des Streits, ist es von ausschlaggebender Bedeutung, ob für England im Zuge des weltweiten Dekolonialisierungsprozesses eine Pflicht zur Aufgabe der territorialen Souveränität besteht.

\section{Die Existenz einer Rechtspflicht zur Dekolonisierung}

Dazu ist zunächst zu prüfen, ob das inder UN Resolution 1514 (XV), der "Declaration on the granting of independence to colonial countries and peoples" vom 14.12.1960 ausgedrückte Dekolonialisierungsgebot, die über die einer politischen Maxime hinausgehende Oualitaet eines Völkerrechtlichen Rechtsinstituts hat, Die Satzung der UNO bietet keine Rechtsgrundlage für ein solches Gebot ${ }^{26}$, auch den UN Resolutionen zu dieser F'rage fehlt nach überwiegender Ansicht die Rechtskraft.

24 Max Huber, a.a.O. s. 35.

25 Verdross-Simma, a.a.O. s. 557.

26 Hier ist lediglich die Verpflichtung zur Entlassung der Mandatsgebiete in die Unabhängigkeit geregelt, Art. 73, 77, UNCharta, vgl. Darstellung bei Hillekamps a.a.O. S. 174 . 
Überzeugend ist dagegen die Begründung eines rechtlich verbindlichen Dekolonisierungsgebotes als Völkergewohnheitsrecht, dem eine entsprechende opinio iuris und eine dieser Rechtsüberzeugung folgende Praxis $\mathrm{zu}$ Grunde liegt, wobei die vergleichsweise kurze Zeitspanne seit Beginn der Aufiösung der Kolonialreiche irrelevant ist ${ }^{27}$.

Einzelne noch verbleibende, meist sehr kleine Kolonialgebiete vermögen die Entstehung einer generellen Rechtspflicht angesichts der überwältigenden Staatenpraxis und der zumẹist einstimmigen Kundgabe dieser Rechtsüberzeugung in den Vereinten Nationen, nicht zu verhindern.

Grossbritannien ist dieser Rechtsüberzeugung durch Auflösung seines Kolonialreiches und entsprechende Erklärungen ebenfalls beigetreten und muss sich diese also gegebenenfalls entgegenhalten lassen.

\section{Liegt auf den Inseln eine koloniale Situation vor?}

Entschieden bestritten wird von England jedoch das Vorliegen einer kolonialen Situation auf den Falklandinseln, von dem die Vereinten Nationen ausgingen, als sie 1963 den Archipel in die Liste der Kolonialländer aufnahmen und den Streit 1964 dem Sonderkommitee für Dekolonialisierung vorlegten.

Ihre Begründung findet diese Auffassung in dem Prinzip IV des Annexes zu der UN Resolution 1514 (XV), Nach dem ein Land, das von dem es verwaltenden Staat geographisch getrennt und ethnisch und oder kulturell verschieden ist, prima facie als 'non self geverning territory' den Rechtsvorschriften der UNO für zu dekolonisierende Gebiete unterliegt. Hinzu tritt, dass die Inseln fern 'vom Mutterland in einem klassischen Kolonisierungsgebiet der europäischen Mächte liegen und ihre Besiedlung durch europäische Auswanderer in einer Hochzeit der Kolonialreiche dieses Bild bestätigen. Im übrigen besitzen die Inseln auch heute noch den Status einer Kronklonoie in relativer Abhängigkeit zum Mutterland.

Die Existenz einer kolonialen Situation ist mithin trotz britischer Einwände zu bejahen.

\section{Hat das Selbstbestimmungsrecht der Völker die Qualitaet eines Rechtsinstituts}

Das in der UN Resolution 1514 (XV) formulierte Dekolonialisie${ }^{27}$ Seidl-Hohenfeldern Ignaz, Dekolonisierung, Politik und positives Recht, JZ. 1964, S. 489. 
rungsgebot fordert die Kolonialmächte $z u$ einer schnellen und unbedingten Beendigung aller Formen des Kolonialismus durch Verwirklichung des Selbstbestimmungsrechts der Völker auf. Es ist zu prüfen, ob ein Selbstbestimmungsrecht der Völker mit verbindlicher Rechtskraft existiert und wenn ja, ob es im vorliegenden Fall Anwendung findet.

Die in der Vergangenheit lebhaft geführte Diskussion um das Bestehen eines Rechtsinstituts des Selbstbiestimmungsrechts der Völker und nicht nur eines politischen Prinzips, wird von der wohl überwiegenden Meinung heute positiv beantwortet ${ }^{28}$.

Ausgangspunkt der Diskussion war die schwer abzugrenzende Ausgestaltung eines solchen Rechts. Während der Begriff des Volkes noch unbestimmt ist, ist hinsichtlich der Modalitäten heute unbestritten, dass das Selbsbestimmungsrecht ausgeübt werden kann durch Errichtung eines Selbstständigen Staates, freien Anschluss an einen bestehenden Staat und durch freien Übergang in einen anderen politischen Status, jedoch allgemein kein Sezessionsrecht gewährt ${ }^{29}$.

\section{Die Beurteilung des vorliegenden Falles}

Fraglich ist, ob das Selbstbestimmungsrecht von einer der beiden Parteien für sich in Anspruch genommen werden kann.

Argentinien bestreitet ein Recht auf Selbstbestimmung der Falkländer und Hinweis auf Art. 6 der Resolution 1514 (XV) der besagt, dass keine Bestrebungen im Rahmen der Dekolonialisierung zur Verletzung der territorialen Integrität und der staatlichen Souveränität eines Landes führen dürfen. Eine solche Verletzung würde aus argentinischer Sicht aber mit einem Votum der Inselbewohner für England gegeben sein. Indes dürfte diese Argumentation fehlgehen, da nach allgemeiner Auslegung Art. 6 nur die Sicherung der, durch die Dekolonisation neugewonnenen Unabhängigkeit bezwecke ${ }^{30}$. Bedenken an einer Durchsetzbarkeit des Selbstbestimmungsrechts der einen oder der anderen Seite entstehen jedoch aus ganz anderen Überlegungen.

Die Forderung nach Verwirklichung des Selbstbestimmungsrechts der Völker, wie sie in der Dekolonisieungsresolution 1514 (XV) vorgetragen wird, geht von dem Fall aus, dass ein Volk unter Verletzung

\footnotetext{
28 wenn auch z.B. Verdross-Simma, a.a.O. .S. 255, nur von einer lex imperfecta ausgehen.

${ }^{2 \theta}$ Verdross-Simma, a.a.O. S. 253., ff.; Berber, a.a.O. S, $174 f f$.

${ }^{30}$ ausführlich Hillekamps, a.a.O. S. $194 \mathrm{ff}$.
} 
seiner fundamentalen Menschenrechte sich unter fremder Herrschaft und Ausbeutung befindet (Art. 1). Für diesen Fall wird in Art. 2 das Selbstbestimmungsrecht postuliert, frei seinen politischen Status zu bestimmen und frei seine wirtschaftliche, soziale und kulturelle Entwicklung, zu verfolgen.

Ein solcher Fall liegt jedoch weder für Argentinien, das seine Selbstbestimmung schon im 19. Jahrhundert in einer unabhängigen, frei bestimmten Staatsorganisation verwirklicht hat, noch für die Inselbewohner vor, die als britische Staatsangehörige sogar nahdrücklich die Zugehörigkeit zum britischen Staatsverband wünschen.

Zusammenfassend ist hier festzustellen, dass zwar eine rechtlich verbindliche Pflicht zur Dekolonisation von Kolonial gebieten besteht, die auch die Falklandinseln betrifft ${ }^{31}$, die Vorraussetzungen des in der UN Resolution 1514 (XV) aufgezeigten Dekolonislerungsprozesses im vorliegenden Fall aber nicht erfüllt sind.

Diesem Sachverhalt tragen die UN Resolution'en 2065 (XX) und 3160 (XXVIII) Rechnung, indem sie den Fall der.Falklandinseln als atypische Kolonialsituation, die sich im Souvergänitätskonflikt zweier Staaten äussert, verstehen, deren Lösüng nur in einer einverständlichen Entscheidung über die Souvergänität zufinden ist.

Aus diesem Grund fordern sie beide Staaten zu Recht $\mathrm{zu}$ Verhandlungen, nur unter 'Berücksichtigung' der Dekolonisierungsresolution und unter Wahrung der 'Interessen' der Inselbewohner und nicht in direkter Anwendung der Resolution und des Selbstbestimmungsrechts auf ${ }^{32}$. Dennoch ist die Aufforderung an beide Staaten zu Verhandlungen über die Souveränitätsfrage als bindende Verpflichtung zu sehen, auf diesem Wege eine Lösung im Sinne des Dokolonisierungsgebotes herbeizuführen ${ }^{33}$.

\footnotetext{
${ }^{31}$ Hier insoweit anders $\mathrm{H}$. Weber, a.a.O. S. 121, der offensichtlich eine Rechtspflicht zur Dekolonisation nicht aus Völkergewohnheitsrecht, sondern aus der Resolution 1514 (XV) direkt ableitet.

32 anders Hillekamps, a.a.O. S. 180f., für den offensichtlich kein Unterschied des vorliegenden Falls zu dem von der UN Resolution 1514 (XV) als Normalfall angenommenem besteht.

${ }^{33}$ Diese besteht für Argentinien wohl im Sinne einer 'Mitwirkungspflicht'.
} 